\title{
Do Simple Cells in Primary Visual Cortex Form a Tight Frame?
}

\author{
Emilio Salinas \\ Instituto de Fisiología Celular, UNAM, Ciudad Universitaria S/N, 04510 México D.F., \\ México
}

\section{F. Abbott}

Volen Center for Complex Systems and Department of Biology, Brandeis University, Waltham, MA 02254-9110, U.S.A

Sets of neuronal tuning curves, which describe the responses of neurons as functions of a stimulus, can serve as a basis for approximating other functions of stimulus parameters. In a function-approximating network, synaptic weights determined by a correlation-based Hebbian rule are closely related to the coefficients that result when a function is expanded in an orthogonal basis. Although neuronal tuning curves typically are not orthogonal functions, the relationship between function approximation and correlation-based synaptic weights can be retained if the tuning curves satisfy the conditions of a tight frame. We examine whether the spatial receptive fields of simple cells in cat and monkey primary visual cortex (V1) form a tight frame, allowing them to serve as a basis for constructing more complicated extrastriate receptive fields using correlationbased synaptic weights. Our calculations show that the set of V1 simple cell receptive fields is not tight enough to account for the acuity observed psychophysically.

\section{Introduction}

There are a number of parallels between representation and learning in neural networks and methods of functional expansion in mathematical analysis. Populations of neurons with responses tuned to properties of a stimulus may be well suited for approximating functions of stimulus parameters (Poggio, 1990; Poggio \& Girosi, 1990; Girosi, Jones, \& Poggio, 1995). If certain conditions are satisfied, such a population can act as a highly efficient substrate for function representation. Accurate representation of a given function depends critically on the synaptic weights connecting input and output neurons. These weights are often adjusted with a delta-type learning rule that is efficient but not biologically plausible. However, if the tuning curves of the input neurons form what is called a tight frame (Daubechies, Grossmann, \& Meyer, 1986; Daubechies, 1990, 1992), function approximation can be achieved using a more biologically reasonable Hebbian or correlation- 


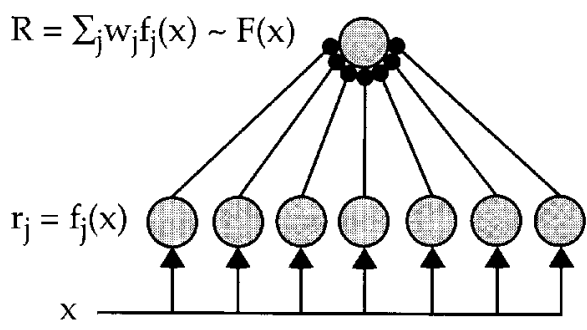

Figure 1: A feedforward network for function approximation. The input neurons at the bottom of the figure respond with firing rates that are functions of the stimulus variable $x$. The firing rate of input neuron $j$ is $f_{j}(x)$. The upper output neuron is driven by the input neurons through synaptic connections; $w_{j}$ represents the strength, or weight, of the synapse from input neuron $j$ to the output neuron. The task is to make the firing rate $R$ of the output neuron approximate a given function $F(x)$.

based rule for the synaptic weights. We investigate here whether the simple cells of primary visual cortex provide such a basis.

To introduce the hypothesis to be tested, we begin with a simple example. Consider a population of $N$ neurons that respond to a stimulus by firing at rates that depend on a single stimulus parameter $x$. The firing rate of neuron $j$ as a function of $x$, also known as the response tuning curve, is $f_{j}(x)$. We wish to examine whether such a population can generate an approximation of another function $F(x)$ that is not equal to any of the input tuning curves. Figure 1 shows a simple network for function approximation. In this network, a population of neurons acting as an input layer drives a single output neuron through a set of synapses. The target function $F$ is represented by the firing rate of the output neuron. The weight of the synapse connecting input neuron $j$ to the output neuron is denoted by $w_{j}$. Following standard neural network modeling practice, we write the firing rate $R$ of the output neuron in response to the input $x$ as a synaptically weighted sum of the input firing rates,

$$
R=\sum_{j=1}^{N} w_{j} f_{j}(x)
$$

If $R=F(x)$, or at least $R \approx F(x)$ to a sufficiently high degree of accuracy, the tuning curve of the output neuron will provide a representation of the target function. The problem is to find a set of input tuning curves, and the synaptic weights corresponding to these tuning curves, that produces an output response close to the target function.

The expansion of a function as a weighted sum of other functions is a standard problem in mathematical analysis. In the limit $N \rightarrow \infty$, any set 
of functions that provides a complete basis over the range of $x$ values considered can be used. Completeness is not very restrictive, but an additional condition is usually applied. The weights $w_{j}$ that make

$$
F(x)=\sum_{j=1}^{\infty} w_{j} f_{j}(x)
$$

can be computed easily if the basis functions $f_{j}$ are orthogonal and normalized so that

$$
\int d y f_{i}(y) f_{j}(y)=\delta_{i j}
$$

where $\delta_{i i}=1$ for all $i$ and $\delta_{i j}=0$ for all $i \neq j$. In this case the weights are given by

$$
w_{j}=\int d y f_{j}(y) F(y)
$$

Equation 1.4 for the weights is closely related to forms of Hebbian and correlation-based learning commonly used in neural networks. A correlationbased rule sets the synaptic weights to values given by

$$
w_{j}=\left\langle f_{j} R\right\rangle,
$$

where the brackets indicate an average over stimuli. Equation 1.5 is equivalent to the statement that the synaptic weight is proportional to the correlation of the firing rates of the pre- and postsynaptic neurons. The idea that synaptic weights are related to correlations in pre- and postsynaptic firing is a standard hypothesis going back to the work of Hebb (1949). Often this principle is expressed in the form of a learning rule that adjusts the weights incrementally toward their desired values during a training period.

We can also think of equation 1.5 as a consistency condition required for the maintenance of weights at their correct values. Suppose that a network has been set up to approximate a given function, so that $R=F(x)$ to a high degree of accuracy. In order for the network to function properly over an extended period of time, some mechanism must maintain the weights at values that produce this desired output. If synaptic weights are maintained by a process that uses a correlation-based rule, condition 1.5 can be interpreted as a consistency condition on maintainable weights. In this interpretation, the Hebbian rule is applied continuously; there is no distinct training period. The average over stimuli in equation 1.5 is then over all stimuli typically encountered, and the output firing rate $R$ can be approximated by the function $F(x)$. Thus, the consistency condition is

$$
w_{j}=\left\langle f_{j}(x) F(x)\right\rangle .
$$


If all stimulus values $x$ occur with equal probability, the average over stimuli is proportional to an integral over $x$, and the weights given by equations 1.4 and 1.6 are proportional to each other. Thus, the Hebbian consistency condition is equivalent to the formula for the coefficients of an orthogonal expansion.

The relationship between equation 1.4 and correlation-based synaptic weights suggests a close relationship between Hebbian ideas about synaptic plasticity and standard mathematical approaches to functional expansion. Unfortunately, a direct link of this type is inappropriate because most sets of neuronal tuning curves are highly overlapping and far from orthogonal. However, all is not lost. What happens if the values of the weights given by equation 1.4 are used in equation 1.1 even when the input tuning functions are not orthogonal? This question can be answered simply by substituting these weights into equation 1.1:

$$
R=\sum_{j=1}^{N} \int d y f_{j}(y) F(y) f_{j}(x) .
$$

If we interchange the order of summation and integration and define

$$
D(x, y) \equiv \sum_{j=1}^{N} f_{j}(x) f_{j}(y),
$$

we find that the output firing rate is a convolution of the target function with $D$,

$$
R=\int d y D(x, y) F(y)
$$

By computing the $D$ function for a given set of input tuning curves, we can determine how accurately a set of neurons can represent a function using synaptic weights given by equation 1.4 . The output firing rate in equation 1.9 will be exactly equal to the target function if $D(x, y)=\delta(x-y)$, since

$$
R=\int d y \delta(x-y) F(y)=F(x),
$$

by the definition of the Dirac $\delta$ function. The condition $D(x, y)=\delta(x-y)$ is less restrictive than the orthogonality condition. A set of functions $f_{j}$ that satisfy $D(x, y)=\delta(x-y)$ is called a tight frame (Daubechies et al., 1986; Daubechies, 1990, 1992).

A finite number of well-behaved tuning curves can never generate a true $\delta$ function in equation 1.8, but they can create a good approximation to one. If the integral of $D(x, y)$ over $y$ is one for all values of $x$ and if 
$D(x, y)$ is significantly different from zero only for values of $y$ near $x$, the integral in equation 1.9 is approximately equal to $F(x)$, provided that $F$ varies more slowly than $D$. Thus, finite sets of tuning curves can act as approximate tight frames. The importance of $D(x, y)$ is that, as a function of $y$, its width determines the resolution with which an output function can be approximated using a correlation rule like equation 1.6 for the synaptic strengths. For this reason, it is interesting to examine $D$ functions for real neuronal tuning curves. The $D$ function provides a useful characterization of the computational capabilities of a population of neurons for function expansion.

To apply ideas about tight frames to real neural circuits, we must find areas where function approximation may be taking place. Primary visual cortex seems to be a good candidate. Neurons in primary visual cortex provide the first cortical representation of the visual world, and their receptive fields provide a basis set from which the more complicated receptive fields of extrastriate neurons are constructed. If the tuning curves of simple cells form a tight frame, the construction of more complicated representations using an architecture like that in Figure 1 can be based on Hebbian synapses whose efficacy is maintained by correlation-based rules of the form given by equation 1.6.

To investigate these ideas, we will compute the $D$ function (and a related function $K$ ) for the receptive fields of simple cells in the primary visual cortices of cats and monkeys. This will allow us to determine how well the receptive field functions of simple cells collectively approximate a tight frame. Lee (1996) has explored the conditions under which sets of filters with properties like those of simple cells satisfy the mathematical definition of a tight frame. We will use the experimentally measured filter distributions to determine explicitly the resolution with which simple cells can generate arbitrary visual filters for downstream neurons using correlation-based synaptic weights.

\section{Function Approximation by Simple Cells}

Representation of the visual world is more complicated than the simplified scenario described in section 1, so we start by recasting the basic problem in terms that are appropriate for this application. Simple cell responses can be expressed approximately through spatiotemporal linear filters acting on visual image intensity, but we will focus exclusively on the spatial aspects of these responses. If $I(\mathbf{x})$ is the luminance (relative to background) of an image that appears at the point $x$ on the retina, where $x$ is a two-dimensional vector, the firing rate of simple cell $j$ is approximated as

$$
r_{j}=g \int d \mathbf{x} f_{j}(\mathbf{x}) I(\mathbf{x})
$$


where $g$ is an overall scale factor determining the magnitude of the firing rate. Because this expression can be positive or negative, we must interpret it as the difference between the firing rates of two neurons having receptive field filters $+f_{j}$ and $-f_{j}$.

The filter functions $f_{j}$ for simple cells are often fit using plane waves multiplied by gaussian envelopes, known as Gabor functions (Daugman, 1985; Jones \& Palmer, 1987; Webster \& De Valois, 1985),

$$
f_{j}(\mathbf{x})=\frac{1}{2 \pi \sigma_{j}^{2}} \exp \left(-\frac{\left|\mathbf{x}-\mathbf{a}_{j}\right|^{2}}{2 \sigma_{j}^{2}}\right) \cos \left(\mathbf{k}_{j} \cdot \mathbf{x}-\phi_{j}\right) .
$$

These filters are parameterized by the receptive field center a, receptive field envelope width $\sigma$, preferred spatial frequency $k \equiv|\mathbf{k}|$, preferred orientation $\theta$ (the direction perpendicular to $\mathbf{k}$ ), and spatial phase $\phi$. For simplicity, we restrict ourselves to circular rather than elliptical receptive field envelopes. We have verified that for values in the physiologically measured range, elliptical receptive fields give essentially the same results reported below for circular receptive fields. We have included a factor of $1 / \sigma^{2}$ in the normalization of the Gabor function so that neurons with different filters give similar maximum firing rates when the optimum images are presented with a given overall amplitude.

In the case of visual responses, the filter functions fill the role that the tuning curves played in the example given in section 1. Similarly, the target function we wish to represent in this case is a filter function. In other words, we attempt to construct a downstream neuron that is selective for some target function or target image $F(\mathbf{x})$. The target response is given by

$$
R=G \int d \mathbf{z} F(\mathbf{z}) I(\mathbf{z}),
$$

with $G$ the scale factor for the output rate. For fixed image power, the maximum value of $R$ is evoked when $I$ is equal to the target function $F$. This output neuron is driven, through synaptic weights, by the responses of a population of simple cells, so that

$$
R=\sum_{j=1}^{N} w_{j} r_{j}=g \sum_{j=1}^{N} w_{j} \int d \mathbf{x} f_{j}(\mathbf{x}) I(\mathbf{x}),
$$

where we have used equation 2.1. Again we impose the condition that synaptic weights are equal to the average product of pre- and postsynaptic firing rates, assuming that the postsynaptic neuron fires as prescribed by equation 2.3. The weights are then given by

$$
w_{j}=\left\langle r_{j} R\right\rangle=G g \int d \mathbf{y} d \mathbf{z} f_{j}(\mathbf{y}) F(\mathbf{z})\langle I(\mathbf{y}) I(\mathbf{z})\rangle .
$$


In this case the average over stimuli, denoted by angle brackets, is an average over the ensemble of visual images. Substituting these weights into equation 2.4 , we find

$$
R=G g^{2} \int d \mathbf{x} d \mathbf{z} K(\mathbf{x}, \mathbf{z}) F(\mathbf{z}) I(\mathbf{x}),
$$

with

$$
K(\mathbf{x}, \mathbf{z})=\int d \mathbf{y} D(\mathbf{x}, \mathbf{y})\langle I(\mathbf{y}) I(\mathbf{z})\rangle
$$

and

$$
D(\mathbf{x}, \mathbf{y})=\sum_{j=1}^{N} f_{j}(\mathbf{x}) f_{j}(\mathbf{y}) .
$$

Equation 2.6 tells us that the firing rate of the output neuron is proportional to a filtered integral of the image,

$$
R=G g^{2} \int d \mathbf{x} F_{e f f}(\mathbf{x}) I(\mathbf{x}) .
$$

The effective filter $F_{\text {eff }}$ is a smeared version of the desired filter $F$ and is given by

$$
F_{e f f}(\mathbf{x})=\int d \mathbf{y} K(\mathbf{x}, \mathbf{y}) F(\mathbf{y}) .
$$

The condition for perfect function representation is then $K(\mathbf{x}, \mathbf{y})=\delta(\mathbf{x}-\mathbf{y})$, and the resolution with which a given filter function can be represented is related to the degree to which $K$ approximates a $\delta$ function.

To determine how well simple cells approximate a tight frame, we first compute both the $D$ and $K$ functions. An interesting aspect of the computation of $D$ from equation 2.8 is that it involves not only the form of the receptive field filters of simple cells, but also the distribution of their parameters across the population of cortical neurons. Once $D$ is determined, we compute $K$ from equation 2.7 by including the correlation function $\langle I(\mathbf{y}) I(\mathbf{z})\rangle$ for natural images (Field, 1987; Ruderman \& Bialek, 1994; Dong \& Atick, 1995). Then we discuss whether $D$ and $K$ behave like a $\delta$ function.

\section{Calculation of $D(\mathbf{x}, \mathbf{y})$}

Equation 2.8, which defines $D$, is a sum over simple cell filters. To compute it, we reparameterize the filters 2.2 in terms of quantities whose distributions 
have been measured. We then approximate the sum over cells by integrals over those parameters using mathematical fits to reported distributions.

To simplify the calculation, we group the simple cell filters into pairs with spatial phases 90 degrees apart (quadrature pairs), corresponding to sine and cosine terms. Each pair of filters can then be described by a single complex exponential filter $g_{j}$,

$$
g_{j}(\mathbf{x})=\frac{1}{2 \pi \sigma_{j}^{2}} \exp \left(-\frac{\left|\mathbf{x}-\mathbf{a}_{j}\right|^{2}}{2 \sigma_{j}^{2}}\right) \exp \left(i\left(\mathbf{k}_{j} \cdot \mathbf{x}-\phi_{j}\right)\right),
$$

where $i \equiv \sqrt{-1}$. Neurophysiological experiments have found pairs of adjacent simple cells roughly 90 degrees out of phase with each other (Pollen \& Ronner, 1981), and we assume that combinations as in equation 3.1 can be formed for all cells. Using complex exponential filters, the sum that defines $D$ becomes

$$
\begin{aligned}
D(\mathbf{x}, \mathbf{y}) & =\sum_{j=1}^{N} g_{j}(\mathbf{x}) g_{j}^{*}(\mathbf{y}) \\
& =\sum_{j=1}^{N} \frac{1}{4 \pi^{2} \sigma_{j}^{4}} \exp \left(-\frac{\left|\mathbf{x}-\mathbf{a}_{j}\right|^{2}+\left|\mathbf{y}-\mathbf{a}_{j}\right|^{2}}{2 \sigma_{j}^{2}}\right) \exp \left(i \mathbf{k}_{j} \cdot(\mathbf{x}-\mathbf{y})\right),
\end{aligned}
$$

where $g_{j}^{*}$ denotes the complex conjugate of $g_{j}$. Note that the spatial phase cancels out in the expression for $D$.

Simple cell receptive field filters are characterized by location, envelope width, and preferred spatial wavelength, phase, and orientation. The envelope width and preferred spatial frequency are correlated; larger receptive fields tend to have lower preferred spatial frequencies. To eliminate this dependence, we will characterize the receptive fields by bandwidth rather than by envelope width. The band width is the width of the spatial frequency tuning curve measured in octaves. It is defined as $\beta \equiv \log _{2}\left(\omega_{2} / \omega_{1}\right)$, where $\omega_{2}$ and $\omega_{1}$ are the higher and lower spatial frequencies, respectively, at which the neuron gives half of its maximum response. The bandwidth is related to the envelope width $\sigma$ and the preferred frequency $k$ by

$$
\sigma=\frac{\sqrt{2 \ln (2)}}{k} \frac{2^{\beta}+1}{2^{\beta}-1} .
$$

We use $\beta$ and $k$ as independent parameters, with the tuning curve width $\sigma$ given by equation 3.3 .

To compute $D$, we use an integral over receptive field parameters as an approximation to the sum over neurons, making the replacement

$$
\sum_{j=1}^{N} \rightarrow \int d \mathbf{a} d k d \beta d \theta d \phi \rho_{a}(\mathbf{a}) \rho_{k}(k) \rho_{\beta}(\beta) \rho_{\theta}(\theta) \rho_{\phi}(\phi),
$$


where the different $\rho$ functions are the neuronal coverage densities (the number of neurons per unit parameter range) for the different receptive field parameters. The validity of this approximation is addressed at the end of this section. Preferred orientations and spatial phases appear to be uniformly distributed over neurons in primary visual cortex (Hubel \& Wiesel, 1974; Field \& Tolhurst, 1986; Bartfeld \& Grinvald, 1992; Bonhoeffer \& Grinvald, 1993) so we assume that $\rho_{\theta}(\theta)$ and $\rho_{\phi}(\phi)$ are constants. Over a small region of the cortex, $\rho_{a}(\mathbf{a})$ can also be approximated as a constant. This means that we are considering a small enough region of the visual field so that the dependence of the cortical magnification factor on eccentricity can be ignored. Since the $D$ function we compute will be quite narrow, this is a reasonable approximation. With these assumptions, we find

$$
\begin{aligned}
D(\mathbf{x}, \mathbf{y})= & \int d \mathbf{a} d k d \beta d \theta d \phi \rho_{k}(k) \rho_{\beta}(\beta) \\
& \left.\frac{c}{\sigma^{4}} \exp \left(\frac{|\mathbf{x}-\mathbf{a}|^{2}+|\mathbf{y}-\mathbf{a}|^{2}}{2 \sigma^{2}}\right) \times p(i k|\mathbf{x}-\mathbf{y}|) \cos (\theta)\right) .
\end{aligned}
$$

In this and all the following expressions, $c$ denotes a constant that absorbs all of the factors that affect the amplitude but not the functional form of $D$. To simplify the above expression, we have made a shift in the definition of the angle $\theta$, which is allowed because the $\theta$ integral is over all values.

The integrals over a and $\phi$ in equation 3.5 can be done immediately to obtain

$$
\begin{aligned}
D(|\mathbf{x}-\mathbf{y}|)= & \int d k d \beta d \theta \rho_{k}(k) \rho_{\beta}(\beta) \\
& \frac{c}{\sigma^{2}} \exp \left(\frac{|\mathbf{x}-\mathbf{y}|^{2}}{4 \sigma^{2}}\right) \operatorname{xp}(i k|\mathbf{x}-\mathbf{y}| \cos (\theta)) .
\end{aligned}
$$

Note that $D$ depends on only the magnitude of $\mathbf{x}-\mathbf{y}$.

To proceed further, we need to determine the density functions $\rho_{k}(k)$ and $\rho_{\beta}(\beta)$. We have done this by fitting three different data sets. We first performed fits on the data reported by Movshon, Thompson, and Tolhurst (1978) for neurons with 5 degree eccentricity or less in cat area 17. The data and fits are shown in Figure 2 (top). The mathematical fits are given by

$$
\rho_{\beta}(\beta)=\frac{16.6}{\sqrt{2 \pi} 0.44} \exp \left(-\frac{(\beta-1.39)^{2}}{2(0.44)^{2}}\right)
$$

and

$$
\rho_{k}(k)=\frac{1.7 k^{2}}{1+(k / 5.2)^{5}},
$$



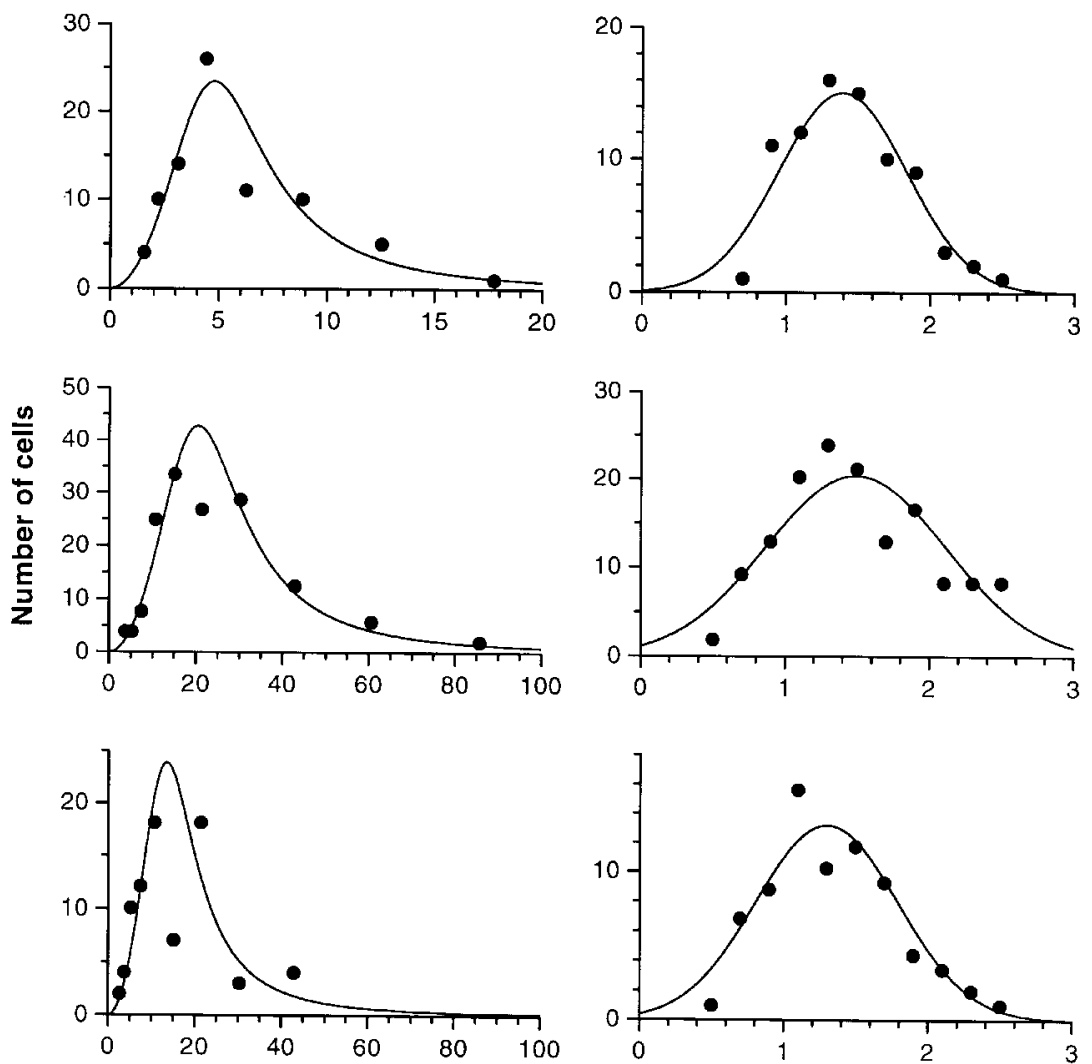

Optimal spatial frequency $(\mathrm{rad} / \mathrm{deg})$

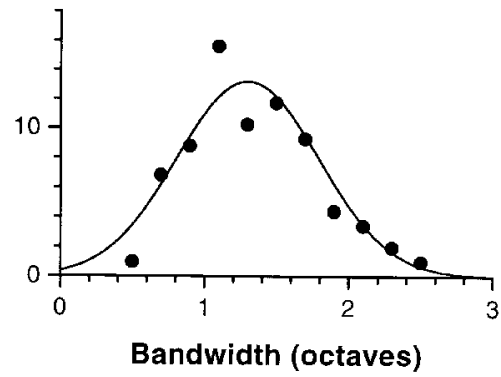

Figure 2: Distributions of optimal spatial frequencies (left) and bandwidths (right) for simple cells. The black circles correspond to experimental data, and the solid curves are mathematical fits. (Top) Cat area 17 simple cells with eccentricities smaller than 5 degrees. Data from Movshon et al. (1978). (Middle) Monkey V1 simple cells within 1.5 degrees of the fovea. Data from De Valois et al. (1982). (Bottom) Monkey V1 simple cells with eccentricities from 3 to 5 degrees (parafoveal sample). Data from De Valois et al. (1982). Notice the different scales in the frequency axes of cat and monkey data. Parameters in the fitting equations were found by minimizing the $\chi^{2}$ statistic (Press et al., 1992). All fits had $P>0.3$, except for the preferred frequency distribution in the monkey parafoveal sample (lower left), which had $P>0.01$.

and we assume that $\rho_{k}(k)=0$ for $k>20$ radians per degree. We also fit two data sets of De Valois, Albrecht, and Thorell (1982) from area V1 in macaque monkeys. These correspond to a region from 0 to 1.5 degrees eccentricity 
(foveal) and another from 3 to 5 degrees eccentricity (parafoveal). The fits for the region closest to the fovea are shown in Figure 2 (middle), and are given by

$$
\rho_{\beta}(\beta)=\frac{31.7}{\sqrt{2 \pi} 0.62} \exp \left(-\frac{(\beta-1.49)^{2}}{2(0.62)^{2}}\right)
$$

and

$$
\rho_{k}(k)=\frac{0.17 k^{2}}{1+(k / 22.2)^{5}} .
$$

We assume that $\rho(k)=0$ for $k>90$ radians per degree. The fits for the parafoveal region are shown in Figure 2 (bottom) and are given by

$$
\rho_{\beta}(\beta)=\frac{16.2}{\sqrt{2 \pi} 0.49} \exp \left(-\frac{(\beta-1.3)^{2}}{2(0.49)^{2}}\right)
$$

and

$$
\rho_{k}(k)=\frac{0.22 k^{2}}{1+(k / 14.6)^{5}} .
$$

Here we assume that $\rho(k)=0$ for $k>50$ radians per degree.

The distributions we have extracted exhibit an interesting property: the bandwidth distributions from all three data sets are very similar, and the preferred spatial frequency distributions are scaled versions of each other to a high degree of accuracy. Because the receptive field width $\sigma$ is inversely proportional to the preferred spatial frequency $k$ (see equation 3.3), the integrand in equation 3.6 depends on $|\mathbf{x}-\mathbf{y}|$ only through the combination $k|\mathbf{x}-\mathbf{y}|$. This means that scaling the distribution $\rho_{k}(k)$ by a factor $s$ will have the effect of scaling the argument of the resulting $D$ function to give $D(s|\mathbf{x}-\mathbf{y}|)$. As a result, we can compute the $D$ function for any one of the distribution pairs and determine $D$ for the others simply by scaling the spatial argument of the function. We have verified through numerical integration that the three bandwidth distributions are equivalent and that this approximation is practically exact (compare Figures $3 \mathrm{~A}$ and $4 \mathrm{~A}$ ). We will use the experimental results for cells nearest to the fovea in the monkey. The $D$ function we compute and show in the figures corresponds to this case, but the results for the parafoveal region of the monkey can be obtained by broadening these curves by a factor of 1.52 , and for the cat data with a broadening factor of 4.27. Results for any eccentricity in either animal can similarly be obtained by scaling the curves by appropriate factors. 

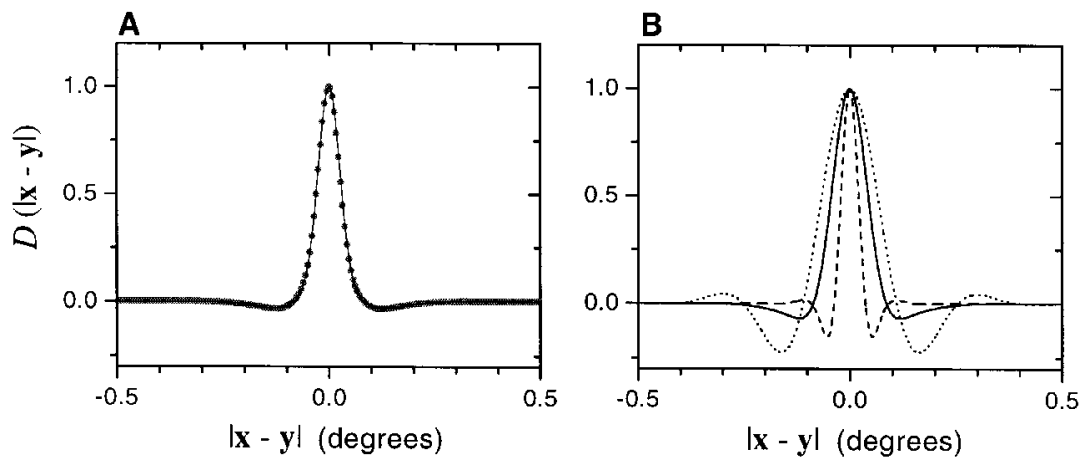

Figure 3: $D$ function for monkey simple cells. All $D$ curves shown are normalized to a maximum height of one. (A) Result when the experimentally measured neuronal distributions, equations 3.9 and 3.10, are used in the calculation of $D$. The filled circles correspond to numerical integration of equation 3.6 for different values of $|\mathbf{x}-\mathbf{y}|$; the continuous line is a fit to the numerical results using a combination of three gaussian functions (see equation 3.13). The width of the curve at half maximum height, which determines the resolution for function approximation, is 0.06 degree. (B) Results obtained when the distributions of bandwidths and preferred frequencies are manipulated. The dotted line is the $D$ function when only neurons near the peaks of both distributions are used; the width at half-height is 0.13 degree. The continuous line is the result when only neurons with optimal frequencies lower than 50 radians per degree are included in the calculation; the width at half-height is 0.086 degree. The dashed line is the result when only neurons with optimal frequencies higher than 50 radians per degree are included; the width at half-height is 0.042 degree. In these last two cases the full bandwidth distribution was used.

Substituting equations 3.3, 3.9, and 3.10 into 3.6, the triple integral can be computed numerically for different values of $|\mathbf{x}-\mathbf{y}|$ to yield the function $D$. The result is shown in Figure $3 \mathrm{~A}$, where $D$ has been normalized to a maximum value of one. The continuous line is a fit to the numerical result, indicated by dots. The fit was obtained by combining three gaussians,

$$
\begin{aligned}
D_{f i t}(|\mathbf{x}-\mathbf{y}|)= & 0.8590 \exp \left(-\frac{|\mathbf{x}-\mathbf{y}|^{2}}{2(0.024)^{2}}\right)-0.3590 \exp \left(-\frac{|\mathbf{x}-\mathbf{y}|^{2}}{2(0.059)^{2}}\right) \\
& -0.2579 \exp \left(\frac{|\mathbf{x}-\mathbf{y}|^{2}}{2(0.085)^{2}}\right)
\end{aligned}
$$

The figure shows that the full width at half-maximum height is 0.06 degrees. It also shows that the sidebands around the central peak are very small; the function does not oscillate. This suggests that $D$ can approximate a $\delta$ function to a resolution of roughly 4 minutes of arc. 

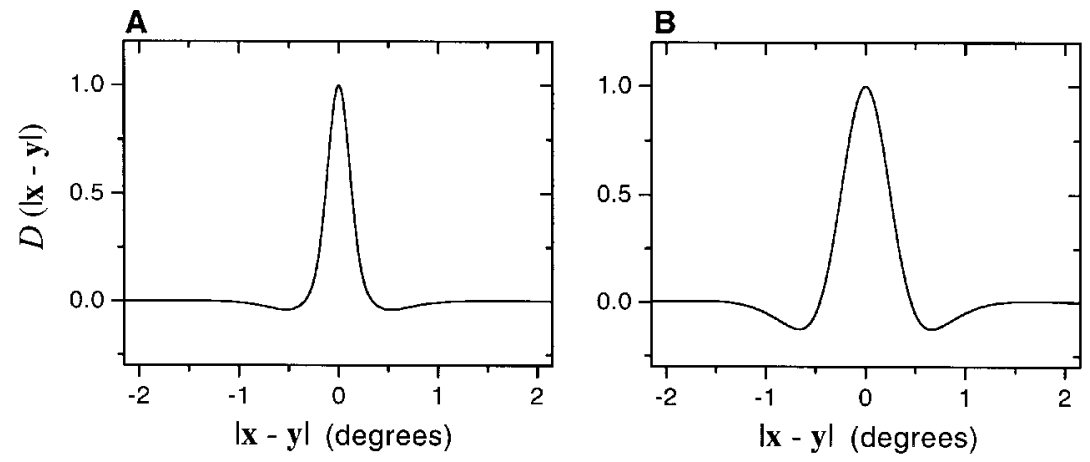

Figure 4: $D$ function for simple cells and retinal ganglion cells of the cat. Both curves are normalized to a maximum height of one. (A) The $D$ function for simple cells of the cat primary visual cortex obtained by numerical integration of equation 3.6. This curve is practically indistinguishable from the result in Figure 3A scaled by a factor of 4.27 . For comparison, the $x$-axes in this figure have been scaled precisely by this amount. The full width at half maximum height is 0.26 degree. (B) The $D$ function for a uniformly distributed population of difference of gaussian filters resembling those of retinal ganglion cells (see the appendix). The full width at half maximum height is 0.5 degree.

In Figure 3B we examine how the $D$ function depends on the distributions of preferred spatial frequencies and bandwidths used in the calculation. First, we investigate what happens if only neurons with parameters near the peaks of the distributions in Figure 2 are considered. We include bandwidths ranging between 1.4 and 1.6 octaves and optimal frequencies ranging between 21 and 23 radians per degree, with no neurons outside these ranges. The $D$ function that results is shown in Figure 3B (dotted line). It has large inhibitory sidebands, and its width at half-height is more than twice the width of the original function. When the full bandwidth distribution is used but the same restricted range of preferred frequencies is included, the result is similar.

The neurons at the tail of the preferred frequency distribution are important in setting the width of $D$, even though proportionally they represent a small fraction of the total. When the distribution is truncated, allowing only optimal frequencies below 50 radians per degree, the resulting $D$ function has a width of 0.086 degrees. Conversely, when only optimal frequencies above 50 radians per degree are included, the resulting $D$ function has a width of 0.042 degrees. Thus, the resolution of the full set of simple cells seems to be limited by the highest frequencies included. The $1 / \sigma^{2}$ factor that appears in equation 2.2 is partly responsible for this effect.

It is interesting to compare the $D$ function of simple cells with that of neurons at earlier stages in the visual pathway. In the case of retinal gan- 
glion cells, the computation is straightforward because their filters can be described by differences of gaussians (see the appendix). The $D$ functions for simple cells and retinal ganglion cells corresponding to data collected in cats are shown in Figure 4. The full widths at half-maximum height are 0.26 and 0.5 degree, respectively. The resolution of simple cells for function approximation in the same animal is better, roughly by a factor of 2 . This is related to the fact that some simple cells have high preferred spatial frequencies or, equivalently, filters that vary more rapidly than those of retinal ganglion cells.

Because the integral in equation 3.6 was computed numerically using the trapezoidal rule (Press, Flannery, Teukolsky, \& Vetterling, 1992), the $D$ function was actually computed as a sum. This is appropriate since the biological system involves individual neurons, not a continuum. The number of terms in the sum is proportional to the number of neurons with receptive fields overlapping the same spatial location. Examining how the number of terms affects the sum reveals how many neurons are needed to obtain a smooth $D$ function. The results shown in Figure 3A were obtained with 30 steps in orientation $\theta$ (in the range $0-2 \pi$ ), 50 steps in preferred frequency $k$ (in the range $0-90$ radians per degree), and 50 steps in bandwidth $\beta$ (in the range 0.1-3.0 radians per degree). With a much coarser grid of 8 orientations, 10 frequencies, and a single bandwidth (same ranges), the resulting curve was almost identical to the one in Figure 3A. In general, the distribution of bandwidths had little impact on the results. Hence, for a given point in visual space, sampling at 8 orientations and 10 frequencies per orientation is enough to construct a $D$ function with a 0.06 degree resolution. Are the neurophysiological data consistent with these requirements? Within the dimensions of a hypercolumn, preferred orientation changes smoothly and uniformly throughout 180 degrees (Hubel \& Wiesel, 1974; Bartfeld \& Grinvald, 1992; Bonhoeffer \& Grinvald, 1993). In fact, in orientation maps obtained with optical recordings, 8 orientation bins in the $0-180$ degrees range can easily be distinguished by eye (Bartfeld \& Grinvald, 1992; Bonhoeffer \& Grinvald, 1993). Thus, sampling in orientation space is not a limitation. On the other hand, the 10 frequency steps do not correspond to 10 neurons, for two reasons. First, each complex filter represents a sine-cosine pair where each element is the difference between $+f_{j}$ and $-f_{i}$, which means 4 neurons per filter. Second, $\rho_{k}(k)$ is not uniform. To get at least 4 complex filters with optimal frequencies inside the 90 radians per degree bin, the 10 frequency bins must involve a total of about 300 filters, or 1200 neurons. This means that for each point in space, at least 1200 frequencies are needed in each of the orientation bins to achieve the resolution implied by the $D$ function. De Valois et al. (1982) estimated that in monkeys, on the order of 100,000 neurons respond to stimuli at a given location in visual space. Assuming that half of those are simple cells and that frequencies are uniformly distributed in the 8 orientation bins (De Valois et al., 1982; Field \& Tolhurst, 1986; Hübener, Shoham, Grinvald, \& Bonhoeffer, 1997) leaves about 6000 frequencies per 
orientation bin. This number, although only a rough figure, suggests that the sampling density in spatial frequency is sufficient to achieve the resolution implied by the $D$ functions that we have computed.

\section{Calculation of $K(\mathbf{x}, \mathbf{y})$}

To compute the function $K$ from $D$, we need to include the two-point correlation function of ensembles of natural images, $Q \equiv\langle I(\mathbf{x}) I(\mathbf{y})\rangle$. This function has been characterized in the frequency domain (Field, 1987; Ruderman \& Bialek, 1994; Dong \& Atick, 1995), so we will use its Fourier transform $\tilde{Q}$ to compute the convolution 2.7 through which the $K$ function is defined (Bracewell, 1965).

Using a minimal set of assumptions, Dong \& Atick (1995) derived an analytic expression for the full spatiotemporal power spectrum of natural images and fit it to data from movies and video recordings. Taking $k$ as the spatial frequency, in radians per degree, and $\omega$ as the temporal frequency, in radians per second, they proposed that

$$
\tilde{Q}(k, \omega)=\frac{A}{k^{m-1} w^{2}} \int_{r_{1} \omega / c k}^{r_{2} \omega / c k} d v v P(v),
$$

where $A$ is a constant that absorbs the amplitude factors, $c=360 / 2 \pi, m$ has been measured from snapshot images to be about $2.3, r_{1}$ and $r_{2}$ are, respectively, the minimum and maximum viewing distances at which objects are resolved, and $P(v)$ is the probability distribution of velocities of objects that are seen. Dong \& Atick (1995) suggested that

$$
P(v) \sim \frac{1}{\left(v+v_{0}\right)^{n}}
$$

which fit their two data sets extremely well using $n=3.7, v_{0}=1.02$ meters per second, $r_{1}$ equal to 2 or 4 meters, and $r_{2}$ either 23 or 40 meters (depending on the data set). The parameter $v_{0}$ is related to the mean speed by $\bar{v}=$ $v_{0} /(n-2)$. We will use the above expression in our calculation of $K$, but to investigate whether the particular choice for $P(v)$ is critical to our results, we also consider another distribution with a different shape:

$$
P(v) \sim v \exp (-\alpha v)
$$

Because we are interested only in the spatial component of $\tilde{Q}(k, \omega)$, we need to average over $\omega$, or consider it as an additional parameter. Given typical ocular movements, a reasonable choice is to set $\omega$ equal to the mean saccade frequency-about 4 per second or $\omega=8 \pi$ radians per second. We will use this value and consider $\tilde{Q}$ as a function of $k$ only. 

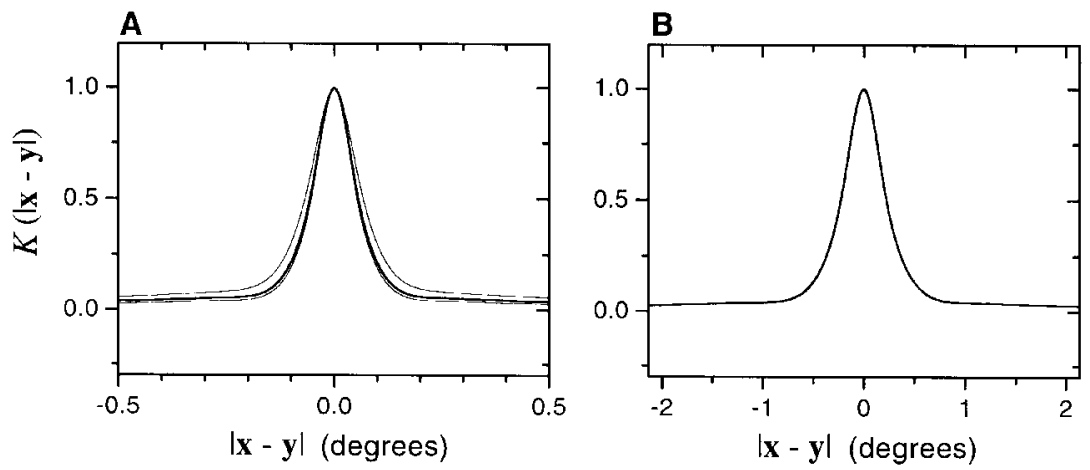

Figure 5: $K$ functions for monkey and cat simple cells. The curves shown correspond to numerical integration of equation 4.5 for different values of $|\mathbf{x}-\mathbf{y}|$. (A) Results for monkey simple cells based on the $D_{\text {fit }}$ function of equation 3.13 and shown in Figure 3A. The thick line is the result when the following parameters were used in equations 4.1 and 4.2: $n=3.7, m=2.3, r_{1}=0.2 \mathrm{~m}, r_{2}=100 \mathrm{~m}$, $v_{0}=1.02 \mathrm{~m} / \mathrm{s}, \omega=8 \pi \mathrm{rad} / \mathrm{s}$. The full width at half height is 0.112 degree. The thin lines were obtained with the same parameters, except $\omega=32 \pi$ (narrower curve; width at half-height is 0.105 degree) and $\omega=2 \pi$ (wider curve; width at half-height is $0.135^{\circ}$ ). (B) Results for cat simple cells also based on $D_{\text {fit }}$ in equation 3.13 , but scaled by a factor of 4.27 . The $x$-axis in this figure was scaled by the same amount. The width at half-height is 0.45 degree.

We first compute the Fourier transform of $K$. This is equal to the product of the Fourier transforms of $D$ and $Q$, since $K$ is defined by a convolution (Bracewell, 1965; Press et al., 1992). We actually use the Fourier transform of $D_{\text {fit }}$, which is simply the sum of the Fourier transforms of three gaussians. The Fourier transform of $K$ is $\tilde{K}=\tilde{D}_{f i t} \tilde{Q}$. Transforming back to the spatial representation we have

$$
K(\mathbf{x}, \mathbf{y})=\frac{1}{4 \pi^{2}} \int d \mathbf{k} \exp (i \mathbf{k} \cdot(\mathbf{x}-\mathbf{y})) \tilde{D}_{f i t}(k) \tilde{Q}(k)
$$

Writing $\mathbf{k}$ in polar coordinates and shifting the angle variable, we obtain

$$
K(|\mathbf{x}-\mathbf{y}|)=\frac{1}{4 \pi^{2}} \int d k d \theta k \exp (i k|\mathbf{x}-\mathbf{y}| \cos (\theta)) \tilde{D}_{f i t}(k) \tilde{Q}(k)
$$

Given values for the parameters of $\tilde{Q}$, the above integral can be computed numerically for each value of $|\mathbf{x}-\mathbf{y}|$. Figure 5 shows the results for the data collected in monkeys (see Figure 5A) and cats (see Figure 5B) integrating equation 4.1 using equation 4.2. For the monkey data, equation 3.13 was used for $D_{f i t}$, and for the cat data this same curve was scaled by a factor of 
4.27. Comparison between Figures $5 \mathrm{~A}$ and $5 \mathrm{~B}$ shows that the $K$ functions are approximately scaled versions of each other; the $K$ function for cats is just slightly narrower than predicted by scaling. The thick curve in Figure 5A is the result for a standard set of parameters, whose values are listed in the caption of Figure 5. The full width at half-height of the curve is 0.112 degree, which is about twice the width of the original $D$ function. Thus, averaging over images worsens the resolution. The thin curves in the same figure were obtained by multiplying the standard value of $\omega$ by $1 / 4$ (wider curve) and by 4 (narrower curve). This has the same effect as multiplying $r_{1}$ and $r_{2}$ or $v_{0}$ by these same factors, because these parameters multiply or divide $\omega$. The thin curves are quite close to the thick one, which means that the parameters in equations 4.1 and 4.2 have relatively little importance in defining the shape and width of $K$. The precise functional form of $P(v)$ did not seem to be crucial either. When equation 4.3 was used (parameter $\alpha$ was set so that the mean speed was the same as before), the results were indistinguishable from those in Figure 5. It is the tail of $\tilde{Q}$, which goes as $1 / f^{m-1}$, that makes $K$ broader than $D$ and defines its shape; the detailed form of $\tilde{Q}$ near $k=0$, which is where the parameters have an influence, does not seem to matter much.

\section{Assessment of Tightness}

We have compared the computed $D$ and $K$ kernels to a $\delta$ function based only on the width of their central peaks, but the tightness of a set of basis functions can be more easily grasped by plotting the Fourier transform of its associated $D$ function. In this way, frequency ranges in which $\tilde{D}$ is approximately flat can be promptly identified. This is important, because the class of functions that are band-limited to such frequency ranges will be accurately represented by the $f_{j}$ basis, that is, the $f_{j}$ functions will behave as a tight frame for the class of functions whose Fourier transform is zero outside the identified ranges. Flatness is quantified by the ratio $\max (\tilde{D}) / \min (\tilde{D})$, which is commonly used as a rigorous measure of tightness (Lee, 1996).

Figure 6 shows the Fourier transforms of several $D$ and $K$ functions. Because $K$ is related to $D$ through a convolution with the correlation of natural images (see equation 2.7), in all cases $\tilde{K}$ is the product of $\tilde{D}$ times the Fourier transform of the natural image correlation, $\tilde{Q}$. Here $\tilde{D}$ reveals the tightness of the original set of functions, when no averaging over images takes place; in contrast, $\tilde{K}$ reveals the tightness of the set when averaging over multiple images does take place (see section 6). Figures $6 \mathrm{E}$ and $6 \mathrm{~F}$ show an ideal case in which the basis set provides perfect approximation of functions with frequency components below 30 cycles per degree; the associated $\tilde{D}$ function is flat in this range. This corresponds, for example, to a set of wavelets (Daubechies, 1990, 1992). For this set $\tilde{K}$ is equal to $\tilde{Q}$, so Figure $6 F$ simply shows the Fourier transform of the natural image correlation func- 

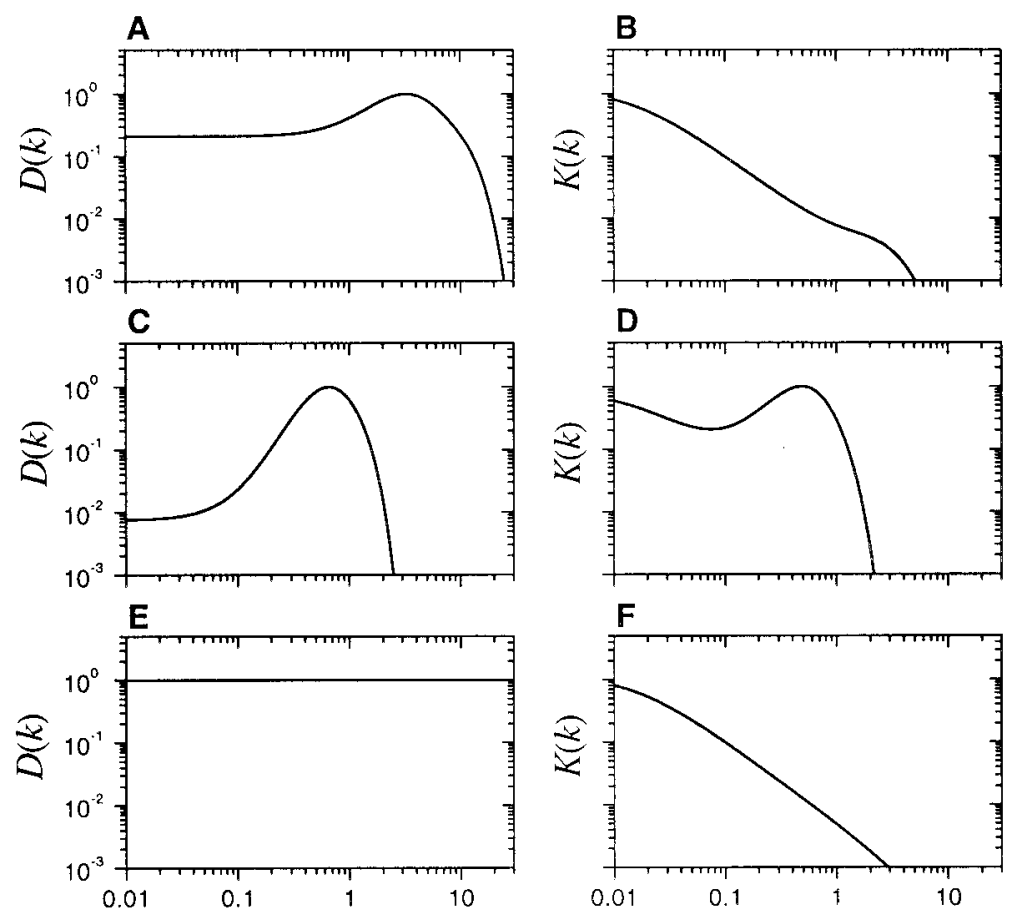

Spatial frequency $k$ (cycles/deg)

Figure 6: Fourier transforms of different $D$ (left) and $K$ (right) functions. In all cases the plots on the right were obtained by multiplying the plots on the left by the Fourier transform of the natural image correlation function, $\tilde{Q}$ (shown in $F$ ). The top row shows the results for monkey $\mathrm{V} 1$ foveal neurons. (A) Fourier transform of the $D$ function shown in Figure 3A. (B) Fourier transform of the $K$ function shown in Figure 5A. The middle row shows the results for retinal ganglion cells of the cat (see the appendix). (C) Fourier transform of the $D$ function shown in Figure 4B. (D) Fourier transform of the $K$ function associated with retinal ganglion cells of the cat. The bottom row shows an ideal case of a set of basis functions whose associated $D$ function is exactly a $\delta$ function. Thus, the $\tilde{K}$ function is equal to $\tilde{Q}$. The $\tilde{Q}$ and $\tilde{D}$ curves were scaled to a maximum of one. Note that in these plots the units of spatial frequency are cycles per degree.

tion. This function is not at all flat; although it looks fairly narrow in x space (not shown, but very similar to Figure 5A), its slowly decaying tail makes it a poor approximation to a $\delta$ function. Figures $6 \mathrm{~A}$ and $6 \mathrm{~B}$ show the results for monkey V1 simple cells (foveal). The $\tilde{K}$ function is very similar to $\tilde{Q}$, suggesting that the ensemble of V1 neurons is not compensating for the average over natural images. On the other hand, $\tilde{D}$ is flat up to about 1 cycle per degree, in- 
dicating that simple cell receptive fields provide an excellent approximation of a tight frame for functions with frequency cutoffs no higher than 1 cycle per degree. Even up to 10 cycles per degree, where simple cell responses start to attentuate significantly, $\tilde{D}$ varies only by a factor between 4 and 5 , not too far from the factor of 2 or less that corresponds to a standard definition of a tight frame (Lee, 1996). As a comparison, Figures 6C and 6D show the results for retinal ganglion neurons from the cat (see the appendix). In this case $\tilde{K}$ is much flatter than $\tilde{D}$, albeit with a cutoff between 1 and 2 cycles per degree.

\section{Discussion}

We have evaluated the capacity of populations of simple cells in primary visual cortex to provide a basis for constructing more complicated receptive fields through Hebbian synaptic weights. There are some similarities between the conditions we have studied, in particular, the suggestion that $K$ is proportional to a $\delta$ function, and the work of Atick and Redlich (1990, 1992) concerning ideas proposed by Barlow (1989) regarding redundancy in neuronal representations. However, there are substantial differences between our approach and theirs. Atick and Redlich (1990, 1992) imposed translational invariance so that all of the neurons they considered had the same receptive field structure. Their formulations resulted in consideration of functions such as $K$, including averages over natural images. Because all the receptive fields were taken to be the same, this approach generated an optimality condition on a single receptive field. Atick and Redlich (1990, 1992) computed several optimal receptive field filters, among them those that solved the equation for $K$ close to a $\delta$ function. The case of function approximation that we consider involves cells with different receptive field structures and depends critically on the distribution of their parameters across the cortical population. Our $D$ and $K$ functions characterize the capacity of the ensemble of all simple cells for function approximation; they are not a property of single cells, as in the work of Atick and Redlich. It is interesting that both cases involve averages over natural images and some similar mathematical expressions, but the quantities being considered are very different: the capacity to transmit information in one case and the accuracy for function approximation in the other.

From the $\tilde{D}$ and $\tilde{K}$ curves computed (see Figure 6), we conclude that the set of monkey V1 simple cells approximates a tight frame with a resolution, given by the cutoff in $\tilde{D}$, on the order of $1 / 10$ of a degree. The resolution for cats, obtained by appropriate scaling of $D$, is about 0.43 degrees. In both cases the $D$ function is more similar to the ideal $\delta$ function than the $K$ function, which includes corrections for natural image statistics. Thus, we find no indication that this basis set is counteracting the effects of averaging over many images. In contrast, sets of filters like those of retinal ganglion neurons seem to be better suited for function approximation when the ef- 
fect of image correlations is included. However, their resolution is rather poor - on the order of 1 degree. We should stress that these resolutions are meaningful only for synaptic modification processes based on correlational mechanisms. In particular, our results do not imply that simple cells have a higher overall resolution than retinal ganglion cells, an impossibility given that retinal ganglion cells provide the input to simple cells. Our results imply only that simple cells serve as a better basis from which neurons selective for arbitrary images can be constructed on the basis of linear superposition and Hebbian synaptic modification.

To understand the difference between using the $D$ and $K$ functions to determine whether simple cell receptive fields form a tight frame, we might imagine two forms of Hebbian learning. For the type of Hebbian learning we discussed in section 1 , synaptic adaptation occurs slowly while an ensemble of images is displayed. In this case, the function $K$ sets the resolution for function approximation with Hebbian synapses. Suppose instead that Hebbian modification of synapses occurs rapidly and only when an image equal to the target function appears. In this case the process is fast enough that images different from the target function do not affect the synaptic weights. For this learning procedure, the resolution for function approximation is set by $D$.

Visual acuity in humans and monkeys, in image discrimination or recognition tasks that involve the activities of large numbers of neurons, is about 1 minute of arc, or 0.017 degree (Westheimer, 1980). This is about six times better than the resolution we have estimated for approximation of an arbitrary function based on $D$. It might seem odd that our estimation is considerably below psychophysical discrimination thresholds, but our analysis applies specifically to single neurons downstream from V1 that combine their inputs in a perfectly linear fashion. The discrepancy indicates that the visual system does not rely exclusively on the tightness of the V1 neuronal ensemble to achieve the acuity exhibited at the behavioral level; additional mechanisms beyond linear summation are required. There are two prominent possibilities for this. First, the neural circuitry may be able to exploit some of its nonlinearities to increase accuracy. On the other hand, higher accuracy may also be achieved through a population of neurons. Considering the ubiquity of population codes throughout the cortex, the capacity of V1 simple cells to act as a basis for Hebbian-based function approximation by single postsynaptic neurons may appear substantial—only a factor of 6 below behavioral performance. However, to try to answer the question posed in the title, the frame formed by simple cells in primary visual cortex is tighter than would be expected from a random distribution of Gabor filters, but not tight enough to account for behavioral acuity.

\section{Appendix}

Here we compute the $D$ function for retinal ganglion cells (X cells). The firing of these cells is well characterized through a linear filtering operation (af- 
terward rectifying the result to eliminate negative rates), as in equation 2.1. In this case each filter is equal to the difference of two gaussians,

$$
f_{j}(\mathbf{x})=\frac{A_{1}}{2 \pi \sigma_{1}^{2}} \exp \left(-\frac{\left(\mathbf{x}-\mathbf{a}_{j}\right)^{2}}{2 \sigma_{1}^{2}}\right)-\frac{A_{2}}{2 \pi \sigma_{2}^{2}} \exp \left(-\frac{\left(\mathbf{x}-\mathbf{a}_{j}\right)^{2}}{2 \sigma_{2}^{2}}\right) .
$$

We assume that the only parameter that varies across neurons is a, the receptive field location. Values for the rest of the parameters are as follows: $A_{1} / A_{2}=17 / 16$ (Linsenmeier, Frishman, Jakiela, \& Enroth-Cugell, 1982), $\sigma_{1}=0.17666^{\circ}$ and $\sigma_{2}=0.53^{\circ}$ (Peichl \& Wäsle, 1979). The $D$ function is computed as in equation 2.8 , using the above filters. The sum over neurons is approximated by an integral over a assuming that the receptive fields are uniformly distributed, so $\rho(\mathbf{a})$ is a constant. The integrals can then be computed analytically, and the result is

$$
\begin{aligned}
D(|\mathbf{x}-\mathbf{y}|)= & \frac{A_{1}^{2}}{4 \pi \sigma_{1}^{2}} \exp \left(-\frac{(\mathbf{x}-\mathbf{y})^{2}}{4 \sigma_{1}^{2}}\right)+\frac{A_{2}^{2}}{4 \pi \sigma_{2}^{2}} \exp \left(-\frac{(\mathbf{x}-\mathbf{y})^{2}}{4 \sigma_{2}^{2}}\right) \\
& -\frac{A_{1} A_{2}}{4 \pi\left(\sigma_{1}^{2}+\sigma_{2}^{2}\right)} \exp \left(-\frac{(\mathbf{x}-\mathbf{y})^{2}}{2\left(\sigma_{1}^{2}+\sigma_{2}^{2}\right)}\right) .
\end{aligned}
$$

The Fourier transform of this expression is

$$
\tilde{D}(k)=\left(A_{1} \exp \left(-\frac{\sigma_{1}^{2} k^{2}}{2}\right)-A_{2} \exp \left(-\frac{\sigma_{2}^{2} k^{2}}{2}\right)\right)^{2},
$$

where $k$ is in radians per degree.

\section{Acknowledgments}

We thank Sacha Nelson for his helpful advice. This research was supported by the Sloan Center for Theoretical Neurobiology at Brandeis University, the National Science Foundation (DMS-9503261), and the W. M. Keck Foundation.

\section{References}

Atick, J. J., \& Redlich, A. N. (1990). Towards a theory of early visual processing. Neural Comput., 2, 308-320.

Atick, J. J., \& Redlich, A. N. (1992). What does the retina know about natural scenes? Neural Comput., 4, 196-210.

Barlow, H. B. (1989). Unsupervised learning. Neural Comput., 1, 295-311.

Bartfeld, E., \& Grinvald, A. (1992). Relationships between orientation preference pinwheels, cytochrome oxidase blobs and ocular dominance columns in primate striate cortex. Proc. Natl. Acad. Sci. USA, 89, 11905-11909. 
Bonhoeffer, T., \& Grinvald, A. (1993). The layout of iso-orientation domains in area 18 of cat visual cortex: Optical imaging reveals a pinwheel-like organization. J. Neurosci., 13, 4157-4180.

Bracewell, R. (1965). The Fourier transform and its applications. New York: McGraw-Hill.

Daubechies, I. (1990). The wavelet transform, time-frequency localization and signal analysis. IEEE Trans. Information Theory, 36, 961-1004.

Daubechies, I. (1992). Ten lectures on wavelets. Philadelphia: SIAM.

Daubechies, I., Grossmann, A., \& Meyer, Y. (1986). Painless nonorthogonal expansions. J. Math. Phys., 27, 1271-1283.

Daugman, J. G. (1985). Uncertainty relation for resolution in space, spatial frequency, and orientation optimization by two-dimensional visual cortical filters. J. Opt. Soc. Am., 2, 1160-1169.

De Valois, R. L., Albrecht, D. G., \& Thorell, L. G. (1982). Spatial frequency selectivity of cells in macaque visual cortex. Vision Res., 22, 545-559.

Dong, D. W., \& Atick, J. J. (1995). Statistics of natural time-varying images. Network, 6, 345-358.

Field, D. (1987). Relations between the statistics of natural images and the response properties of cortical cells. J. Opt. Soc. Am. A, 4, 2379-2394.

Field, D., \& Tolhurst, D. J. (1986). The structure and symmetry of simple-cell receptive-field profiles in the cat's visual cortex. Proc. R. Soc. Lond. B, 228, 379-400.

Girosi, F., Jones, M., \& Poggio, T. (1995). Regularization theory and neural networks. Neural Comput., 7, 219-269.

Hebb, D. O. (1949). The organization of behavior: A neuropsychological theory. New York: Wiley.

Hubel, D. H., \& Wiesel, T. N. (1974). Sequence regularity and geometry of orientation columns in the monkey striate cortex. J. Comp. Neurol., 158, 267-294.

Hübener, M., Shoham, D., Grinvald, A., \& Bonhoeffer, T. (1997). Spatial relationships among three columnar systems in cat area 17. J. Neurosci., 17, 9270-9284.

Jones, J., \& Palmer, L. (1987). An evaluation of the two-dimensional Gabor filter model of simple receptive fields in cat striate cortex. J. Neurophysiol., 58, 12331259.

Lee, T. S. (1996). Image representation using 2D Gabor wavelets. IEEE Trans. Pattern Analysis and Machine Intelligence, 18, 959-971.

Linsenmeier, R. A., Frishman, L. J., Jakiela, H. G., \& Enroth-Cugell, C. (1982). Receptive field properties of $X$ and $Y$ cells in the cat retina derived from contrast sensitivity measurements. Vision Res., 22, 1173-1183.

Movshon, J. A., Thompson, I. D., \& Tolhurst, D. J. (1978). Spatial and temporal contrast sensitivity of neurons in area 17 and 18 of the cat's visual cortex. J. Physiol., 283, 101-120.

Peichl, L., \& Wäsle, H. (1979). Size, scatter and coverage of ganglion cell receptive field centers in the cat retina. J. Physiol. (Lond.), 291, 117-141.

Poggio, T. (1990). A theory of how the brain might work. Cold Spring Harbor Symposium on Quantitative Biology, 55, 899-910.

Poggio, T., \& Girosi, F. (1990). Regularization algorithms for learning that are equivalent to multilayer networks. Science, 247, 978-982. 
Pollen, D. A., \& Ronner, S. F. (1981). Phase relationships between adjacent simple cells in the visual cortex. Science, 212, 1409-1411.

Press, W. H., Flannery, B. P., Teukolsky, S. A., \& Vetterling, W. T. (1992). Numerical recipes in $C$. New York: Cambridge University Press.

Ruderman, D. L., \& Bialek, W. (1994). Statistics of natural images: Scaling in the woods. Phys. Rev. Let., 73, 814-817.

Webster, M. A., \& De Valois, R. L. (1985). Relationship between spatial-frequency and orientation tuning of striate-cortex cells. J. Opt. Soc. Am. A, 2, 1124-1132.

Westheimer, G. (1980). The eye, including central nervous system control of eye movements. In V. B. Mountcastle (Ed.), Medical physiology (Vol. 1, pp. 481503). St. Louis: Mosby.

Received June 11, 1998; accepted February 4, 1999. 